\title{
Leptin signal transduction underlies the differential metabolic response of LEW and WKY rats to cafeteria diet
}

\author{
N Martínez-Micaelo, N González-Abuín, A Ardévol, M Pinent, E Petretto ${ }^{2,3}$, \\ J Behmoaras' and M Blay \\ MoBioFood Research Group. Department of Biochemistry and Biotechnology, Universitat Rovira i Virgili, \\ Campus Sescelades, C/Marcel.li Domingo, s/n, 43007 Tarragona, Spain \\ ${ }^{1}$ Imperial College London, Centre of Complement and Inflammation Research, Du Cane Road, London W12 0NN, UK \\ ${ }^{2}$ Imperial College London, MRC Clinical Sciences Centre, Hammersmith Hospital, Du Cane Road, \\ London W12 ONN, UK \\ ${ }^{3}$ Duke-NUS Graduate Medical School Singapore, 8 College Road, 169857 Singapore, Republic of Singapore
}

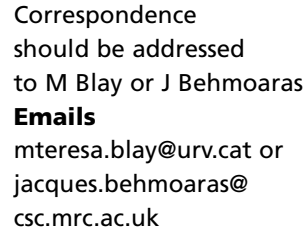

\begin{abstract}
Although the effect of genetic background on obesity-related phenotypes is well established, the main objective of this study is to determine the phenotypic responses to cafeteria diet (CAF) of two genetically distinct inbred rat strains and give insight into the molecular mechanisms that might be underlying. Lewis (LEW) and Wistar-Kyoto (WKY) rats were fed with either a standard or a CAF diet. The effects of the diet and the strain in the body weight gain, food intake, respiratory quotient, biochemical parameters in plasma as well as in the expression of genes that regulate leptin signalling were determined. Whereas CAF diet promoted weight gain in LEW and WKY rats, as consequence of increased energy intake, metabolic management of this energy surplus was significantly affected by genetic background. LEW and WKY showed a different metabolic profile, LEW rats showed hyperglycaemia, hypertriglyceridemia and high FFA levels, ketogenesis, high adiposity index and inflammation, but WKY did not. Leptin signalling, and specifically the LepRb-mediated regulation of STAT3 activation and Socs3 gene expression in the hypothalamus were inversely modulated by the CAF diet in LEW (upregulated) and WKY rats (downregulated). In the present study, we show evidence of gene-environment interactions in obesity exerted by differential phenotypic responses to CAF diet between LEW and WKY rats. Specifically, we found the leptin-signalling pathway as a divergent point between the strain-specific adaptations to diet.
\end{abstract}
Key Words
- cafeteria diet
- inbred rats
- leptin signalling
- LEW
- WKY
- SOCS3

\section{Introduction}

A prolonged excess of nutrient consumption results in the continuous saturation of the white adipose tissue's ability to store energy as fat, triggering a cytokine-driven response that underlie an obesity-induced inflammation.
Thus, white adipose tissue plays a key role in the regulation of metabolism, not only as the major site for energy storage but also as an endocrine organ that regulates the production of hormones such as leptin
Journal of Molecular

Endocrinology

(2016) 56, 1-10 
(Galic et al. 2010, Kanneganti \& Dixit 2012). Although the obesity-induced inflammatory response is triggered and takes place primarily in white adipose tissue, other organs are involved. C-reactive protein (CRP) is an acute-phase protein, which is produced mainly in the liver, and high circulating levels of CRP are considered biomarkers of systemic inflammation in obesity (Fröhlich et al. 2000).

Leptin is produced in proportion to fat stores in order to regulate energy metabolism via its highly expressed cognate receptor in the central nervous system (Myers et al. 2008). In addition to its key role in the maintenance of energy homeostasis, leptin modulates both humoral and cell-mediated immunity (La Cava \& Matarese 2004). Leptin signalling is dependent on the presence of the long isoform of the leptin receptor (LerRb). Leptin stimulation activates the Janus-activated kinase (JAK)/signal transducers and activators of transcription (STAT) pathway, leading to the formation of a LepRb/JAK2 complex that, after trans-phosphorylation, results in STAT3 activation and the induction of SOCS3 expression; SOCS3 in turn negatively regulates leptin signalling by phosphorylating JAK2 (Frederich et al. 1995a, Bjørbæk et al. 1999).

The susceptibility to obesity has a large underlying genetic component, but environmental influences, such as dietary effects, are required to induce its manifestation (Pérusse \& Bouchard 2000, Speakman 2004, Bouchard 2008, Ordovas 2008). Furthermore, although the interactions between genes and the environment that are associated with the development of obesity are not fully understood, studies of inbred animal strains showed susceptibility to diet-induced obesity, whereas other strains show resistance, therefore suggesting that the genetic background contribute to the variability in obesogenic phenotypes between individuals (Hu et al. 2004, Koza et al. 2006, Parks et al. 2013). Diet-induced obesity models, such as the cafeteria (CAF) diet-induced obesity, are considered to be a robust model of the human metabolic syndrome and its related pathologies (Sampey et al. 2011). In a CAF diet, obesity is induced as consequence of hyperphagia resulting from the voluntary intake of highly palatable and energy-dense cafeteria-style foods present in Western-type diets instead of the standard chow (Sampey et al. 2011).

Taking into account the distinct genetic background of inbred rat strains (genetically different) such as Lewis (LEW) and Wistar-Kyoto (WKY) rats, and using the CAF diet-induced obesity model, both genetic and environmental factors can be assessed, providing a powerful tool for understanding the interactions between the genotype and the environment that underlie the development of complex traits such as metabolic syndrome. The WKY and LEW rats share the same MHC haplotype (RT1-1) but show contrasting susceptibility to experimentally induced crescentic glomerulonephritis (Crgn) through macrophage activation. For this reason, these two strains have been used in genome-wide linkage studies aiming to identify susceptibility loci for Crgn (Behmoaras et al. 2008, 2010). In addition, WKY and LEW genomes ( $>20 \times$ coverage) have been sequenced and investigated the transcriptome of their primary macrophages. All these studies showed that these two inbred rat strains show distinct genome and transcriptome profiles and also contrasting macrophage function, which partly explains their susceptibility to Crgn (Maratou et al. 2011, D'Souza et al. 2013).

Here, we studied the phenotypic responses of LEW and WKY rats in response to CAF diet and determined the molecular mechanisms that underlie the metabolic differences between these strains. We found that although the CAF diet promoted higher energy intake and weight gain in both strains, the way this energy excess was metabolically managed was different between WKY and LEW rats, and the metabolic profile was also strainspecific. Finally, our data suggest the differential phenotypic response to CAF diet was due to a differential molecular regulation in the leptin axis between the rat strains.

\section{Materials and methods}

\section{Animals and experimental design}

Male LEW (LEW/Crl) and WKY (WKY/NCrl) (Charles River, Margate, UK) rats, 8 weeks old, were housed individually in a $22{ }^{\circ} \mathrm{C}$ temperature-controlled room with a ratio of $12 \mathrm{~h}$ light: $12 \mathrm{~h}$ darkness cycle with lights on at $0900 \mathrm{~h}$. After an adaptation period, rats from each strain were randomly distributed into two experimental groups $(n=5)$ and fed with either a standard chow diet (STD) (Panlab, Barcelona, Spain) or a cafeteria diet (CAF) composed of bacon, biscuits, pate, muffins, carrots and milk with sugar for seven weeks. The daily total energy content of STD and CAF diets was 2.9 and $7.5 \mathrm{Kcal} / \mathrm{g}$ respectively. The energy distribution of diets were $21.7 \%$ of proteins, $66.2 \%$ of carbohydrates and $9.6 \%$ of lipids in STD diet and $10.4 \%$ of proteins, $38.6 \%$ of carbohydrates and $50.8 \%$ of lipids in CAF diet. After 7 weeks of the indicated diet, the rats were fasted for $9 \mathrm{~h}$ and euthanized by exsanguination under anaesthesia. Blood was collected from abdominal aorta using heparin as anticoagulant.

Published by Bioscientifica Ltd. 
The liver, hypothalamus and white adipose tissue depots (mesenteric, epididymal and retroperitoneal) were weighed, frozen in liquid nitrogen and stored at $-80^{\circ} \mathrm{C}$. All the procedures were approved by the Animal Ethics Committee of the Universitat Rovira i Virgili.

\section{Measurement of adiposity and body weight gain}

The effects of diet on body weight gain were determined by weekly measuring the weight difference between the current week and those weights measured at the beginning of the experiment. The adiposity index was calculated at the end point.

\section{Food intake}

The rats were housed individually in metabolic cages for $48 \mathrm{~h}$, and their food intake was assessed in week 5 of the experiment based on a measurement of the feeding pattern for each animal. The total energy intake and the contribution of each macronutrient to the total intake were normalised to the metabolic mass.

\section{Energy metabolism}

The energy metabolism components were measured, in at least three rats per group, using an indirect open-circuit calorimeter (Panlab Harvard Apparatus, Barcelona, Spain). The oxygen consumption $\left(\mathrm{VO}_{2}\right)$ and carbon dioxide production $\left(\mathrm{VCO}_{2}\right)$ of the rats were monitored simultaneously every $10 \mathrm{~min}$ in two metabolic chambers during the first $200 \mathrm{~min}$ of the postprandial period. The respiratory quotient was calculated from gas exchange measurements $\left(\mathrm{RQ}=\mathrm{VCO}_{2} / \mathrm{VO}_{2}\right)$, and the substrate oxidation values were calculated as previously described (Ferrannini 1988, Arch et al. 2006).

\section{Measurement of biochemical parameters in plasma}

Triglycerides and glucose levels were measured by enzymatic colorimetric methods (QCA, Barcelona, Spain), $\beta$-hydroxybutyrate, non-esterified free fatty acids and leptin were determined by enzymatic colorimetric test kits provided by BEN (Milano, Italy), WAKO (Neuss, Germany) and EMD Millipore (Darmstadt, Germany) respectively.

\section{Quantitative RT-PCR}

Total RNA from the liver, mesenteric fat depot and hypothalamus was isolated using TRIzol reagent according to the manufacturer's instructions (Invitrogen) and purified with RNeasy Mini Kit spin columns (Qiagen). cDNA was generated by using the reverse transcription reagent kit (Applied Biosystems) and was subjected to realtime PCR amplification using SsoFast EvaGreen SuperMix (Bio-Rad). Relative gene expressions were normalised according to the cyclophilin peptidylprolyl isomerase A (Ppia) mRNA levels. The primers sequences used are LepRb5' AGCCCTAGTCGGATCACTCC 3'-F 5' GAGGTTGGATAGGCCAGGTT 3'-R, Lep 5' GATCCTCACCAGCTTGCCTT 3'-F 5' GGGAGCAGCTCTTGGAGAAG 3'-R, Socs3 5' CTGGACCCATTCGGGAGTTC 3'-F 5' CTGGGAGCTACCGACCATTG 3'-R, Crp 5' GGCTTTTGGTCATGAAGACATG 3'-F 5' TCTTGGTAGCGTAAGAGAAGA 3'-R and Ppia 5' CTTCGAGCTGTTTGCAGACAA 3'-F 5' AAGTCACCACCCTGGCACATG 3'-R, 5' - AGGAGCACCCTGAAGCTGA 3'-F 5' TCCTCACATCGGGGAGGTAG 3'-R STAT3. The relative gene expression was normalised to the Ppia mRNA levels using the $2^{-\Delta \Delta C t}$ method.

\section{Statistical analysis}

The phenotypic data are expressed as the mean \pm s.e.m. Weight gain, energy expenditure, RQ and substrate oxidation as parameters measured over a time period were analysed for strain, diet and strain-by-diet interaction using two-way ANOVA for repeated-measures test. Singletime measurements (energy intake, plasma parameters and gene expression) were analysed using standard twoway ANOVA. Differences were considered to be significant when the $P$ values were $<0.05$, and post hoc comparisons were made using a Bonferroni multiple-comparison test. The relationship between variables was assessed by nonparametric Spearman correlations. Calculations were performed using SPSS 17.0 software.

\section{Results}

The cafeteria diet promotes voluntary hyperphagia and weight gain but a differential profile of adiposity in LEW and WKY rats

To investigate the role of the genetic background on the phenotypic responses to diet-induced obesity, two inbred rat strains with distinct genetic backgrounds (PMID: 23890820), LEW and WKY, were fed with a CAF diet for 7 weeks. The rats were allowed free access to highly palatable and energy-dense foods; animals of both genetic backgrounds showed significantly increased body weight in response to CAF diet over time corresponding to an

Published by Bioscientifica Ltd. 
overweight condition. Significant interactions were found between time and both, the type of diet for LEW and WKY rats (factor interaction, $P<0.001$ in all interactions), no interaction was found between time, type of diet and strain altogether (Fig. 1A and B).

This weight gain was accompanied by a significant increase in the total energy intake resulting from voluntary hyperphagia $(168.74 \pm 4.1$ vs $274.31 \pm 5.1 \mathrm{Kcal} /$ day $\times \mathrm{kg}^{0.75}$ in LEW and $177.94 \pm 8.3$ vs $286.92 \pm 9.1 \mathrm{Kcal} /$ day $\times \mathrm{kg}^{0.75}$ in WKY) (Fig. 1C). The CAF diet-induced enhance of energy intake was mainly due to a substantial increase in fat intake (Fig. 1D). No differences were observed between the caloric intake of LEW and WKY (Fig. 1C, D, E and F). When comparing the effects of the CAF diet on the contribution of each macronutrient to the total energy intake, the percentage of calories from fat intake shifted from the least important to the most important energy source, independent of the genetic background (Fig. 1G and H). Therefore, LEW and WKY rats showed a different profile of adiposity induced by the CAF diet (Table 1) and CAF-fed LEW and WKY rats showed greater adipose accumulation than the STD-fed rats.

The content of triglycerides in liver was higher in WKY than in LEW because of the CAF diet $(1.10 \pm 0.075$ vs
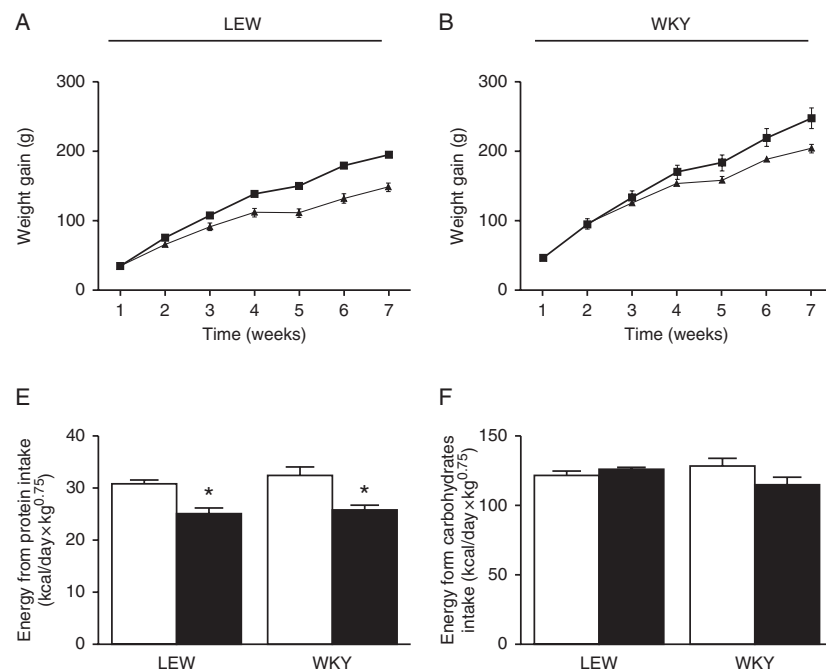

\section{Figure 1}

Body weight and food intake pattern in response to the STD and CAF diets. ( $A$ and B) Body weight gain changes in STD- and CAF-fed LEW and WKY rats respectively (two-way ANOVA for repeated measurements; $n=5 /$ group). ( $C, D, E$ and F) The measured total energy intake (standard two-way ANOVA; $n=5 /$ group, $P<0.001$ for diet, $P=0.162$ for strain and $P=0.819$ for diet and strain interaction) and the energy intake from fat (standard two-way ANOVA; $n=5 /$ group, $P<0.001$ for diet, $P=0.010$ for strain and $P=0.012$ for diet and strain interaction), protein (standard two-way
$2.25 \pm 0.070 \mu \mathrm{g}$ TGs $/ \mathrm{mg}$ liver in LEW and $2.57 \pm 0.4$ vs $3.44 \pm 0.4 \mu \mathrm{g}$ TGs/mg liver in WKY; $P<0.05$ ).

\section{Energy metabolism in response to CAF diet is genetically influenced in the rat}

To investigate the effects of CAF diet-induced overfeeding on the postprandial energy metabolic response, we determined the energy expenditure, metabolic rate and fuel oxidation in LEW and WKY rats.

An increase in energy expenditure was observed during the earliest times of postprandial phase independent of the genetic background and diet; this increase was greater and more sustained for LEW (STD and CAF) rats (Fig. 2). Although the energy expenditure of CAF-fed LEW rats was higher than that of the corresponding STD-fed rats at some specific time points, no significant differences were observed for the postprandial energy expenditure, determined as the area under the curve (AUC) (Fig. 2A). In contrast, for WKY rats, the CAF diet induced a shorter but greater increase in energy expenditure compared to the STD diet, and this expenditure remained high during most of the postprandial state. This resulted in a CAF-induced increase in the energy
C

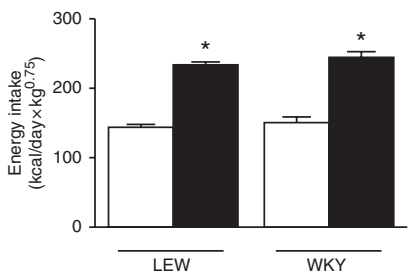

G

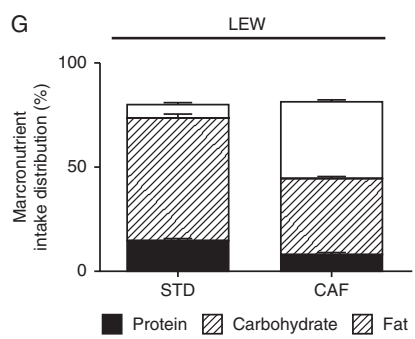

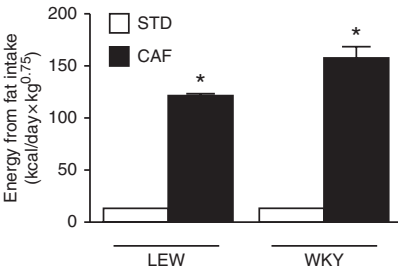

$\mathrm{H}$

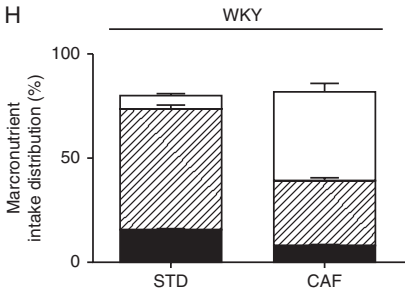

ANOVA; $n=5 /$ group, $P<0.001$ for diet, $P=0.284$ for strain and $P=0.743$ for diet and strain interaction) and carbohydrates respectively (standard two-way ANOVA; $n=5 /$ group, $P=0.331$ for diet, $P=0.630$ for strain and $P=0.065$ for diet and strain interaction). (G and $\mathrm{H}$ ) The distribution of the macronutrient intake based on the food consumption in STD- and CAF-fed LEW and WKY rats respectively. ${ }^{*} P<0.05$ between the rats fed with the STD diet and the CAF diet within each strain, defined as the diet effect.

Published by Bioscientifica Ltd. 
Table 1 The effects of the CAF diet on adiposity and organ weight

\begin{tabular}{l}
\hline Organ weight \\
\hline Metabolic organs \\
Liver weight $(\mathrm{g})$ \\
Kidneys weight $(\mathrm{g})$ \\
Immune-involved organs \\
Spleen weight $(\mathrm{g})$ \\
Thymus weight $(\mathrm{g})$ \\
White adipose tissue depots \\
Mesenteric fat $(\mathrm{g})$ \\
Epididymal fat $(\mathrm{g})$ \\
Perirenal fat $(\mathrm{g})$ \\
Adiposity index $(\%)$ \\
\hline
\end{tabular}

\begin{tabular}{ccc}
\multicolumn{2}{c}{ LEW } \\
\cline { 1 - 1 } Lean & & Cafeteria \\
\cline { 1 - 1 } $11.09 \pm 0.43$ & & $12.35 \pm 0.32$ \\
$2.85 \pm 0.05$ & & $2.97 \pm 0.08$ \\
& & \\
$0.76 \pm 0.06$ & & $0.63 \pm 0.03$ \\
$0.46 \pm 0.02$ & & $0.58 \pm 0.03$ \\
& & \\
$3.93 \pm 0.38$ & & $7.95 \pm 0.47^{*}$ \\
$3.53 \pm 0.16$ & & $11.32 \pm 1.56^{*}$ \\
$4.98 \pm 0.37$ & & $11.37 \pm 1.01^{*}$ \\
$3.05 \pm 0.13$ & & $7.42 \pm 0.64^{*}$ \\
\hline
\end{tabular}

\begin{tabular}{|c|c|}
\hline \multicolumn{2}{|c|}{ WKY } \\
\hline Lean & Cafeteria \\
\hline $7.16 \pm 0.17$ & $8.28 \pm 1.55$ \\
\hline $1.99 \pm 0.07$ & $2.47 \pm 0.07$ \\
\hline $0.61 \pm 0.02$ & $0.59 \pm 0.05$ \\
\hline $0.27 \pm 0.01$ & $0.44 \pm 0.03$ \\
\hline $2.27 \pm 0.18$ & $3.78 \pm 0.49$ \\
\hline $3.22 \pm 0.07$ & $6.98 \pm 0.57 *$ \\
\hline $4.29 \pm 0.31$ & $8.96 \pm 0.41 *$ \\
\hline $3.40 \pm 0.13$ & $5.99 \pm 0.25 *$ \\
\hline
\end{tabular}

$* P<0.05$ vs STD diet-fed rats.

expenditure of WKY rats, measured as the AUC during the postprandial state (Fig. 2B).

The metabolic rate measured as the respiratory quotient (RQ) showed similarly a difference in the energy metabolism pattern between LEW and WKY rats. The STDfed LEW rats maintained a RQ around of 1.0-1.1, which can be associated primarily with carbohydrate oxidation and endogenous fatty acid synthesis (Fig. 2C) (as expected from the predominance of carbohydrates as the main
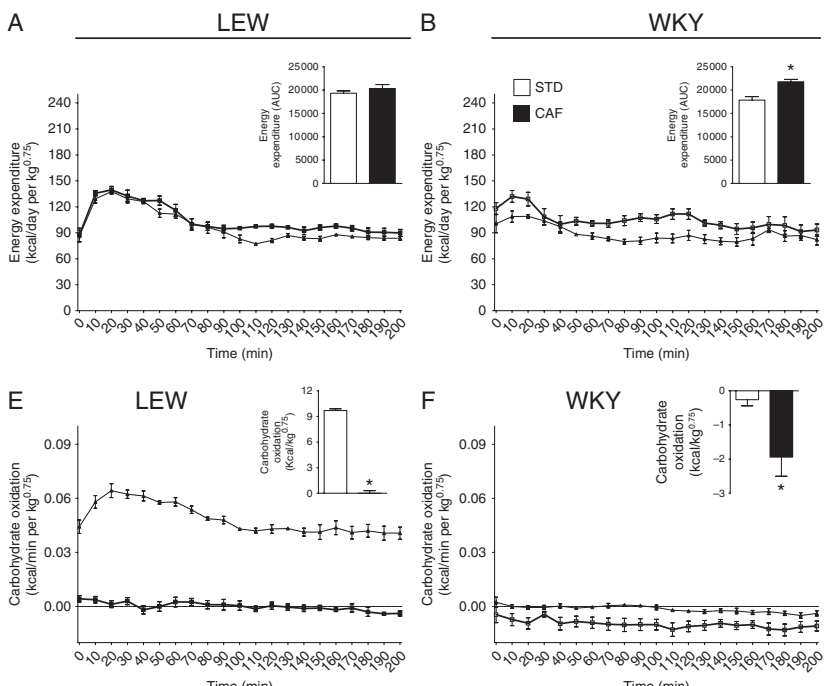

Time (min)

Time (min)

\section{Figure 2}

Postprandial metabolic responsiveness to diet. Energy expenditure, $\mathrm{RQ}$ and substrate oxidation were determined based on gaseous exchange measured by indirect calorimetry during the first $200 \mathrm{~min}$ of the postprandial period in STD- and CAF-fed LEW and WKY rats. ( $A$ and $B$ ) The energy expenditure for LEW and WKY rats (two-way ANOVA for repeated measurements, $P=0.001$ for diet, $P=1.000$ for strain and $P=0.546$ for diet and strain interaction); the inset graphs correspond to the area under the curve (AUC) measured during the 200 min recorded. (C and D) The respiratory quotients of LEW and WKY rats respectively, calculated using

http://jme.endocrinology-journals.org DOI: 10.1530/JME-15-0089
() 2016 Society for Endocrinology Printed in Great Britain
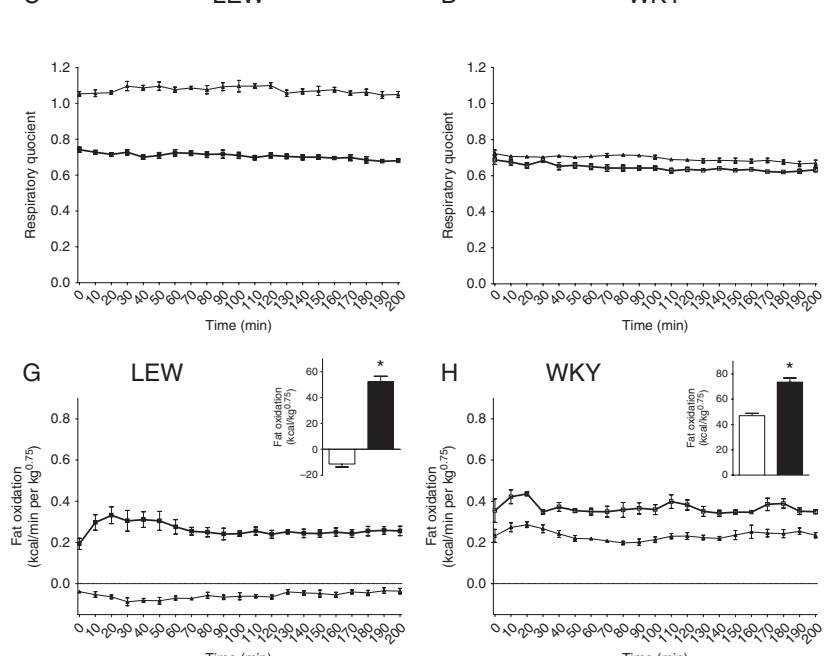

Time (min)

C

Time (min)
D WKY

substrate source (Fig. 2E) instead of the oxidation of fat (Fig. 2G)). However, the RQ determined in STD-fed WKY rats was lower when comparing with STD-fed LEW rats and significantly greater for most measured time points than in CAF-fed WKY rats (Fig. 2D). The RQ of STD-fed WKY rats suggested that fat is the principal fuel for energy production (Fig. 2H) instead of carbohydrates (Fig. 2F), indicating that the energy metabolism pattern of STD-fed WKY rats differed with respect to that observed in STD-fed the quotient $\mathrm{VCO}_{2} \mathrm{NO}_{2}$ (two-way ANOVA for repeated measurements, $P=0.003$ for diet, $P=0.027$ for strain and $P=0.200$ for diet and strain interaction). ( $E$ and $F$ ) Carbohydrate oxidation in response to the diet challenge (STD or CAF diets) for LEW and WKY rats (two-way ANOVA for repeated measurements, $P<0.001$ for diet, $P<0.001$ for strain and $P=0.096$ for diet and strain interaction). ( $G$ and $H$ ) Fat oxidation during the postprandial state after the consumption of the STD or CAF diet in LEW and WKY rats (two-way ANOVA for repeated measurements, $P=0.001$ for diet, $P<0.001$ for strain and $P=1.000$ for diet and strain interaction). 
LEW rats. The CAF-fed LEW and CAF-fed WKY rats showed a RQ of approximately 0.70 (Fig. 2C and D), suggesting that CAF-diet promotes fat oxidation rather than carbohydrate oxidation (Fig. 2E and F) as the most important fuel energetic source (Fig. $2 \mathrm{G}$ and $\mathrm{H}$ ).

\section{The profile of diet-induced circulating metabolites is genetically influenced in the rat}

The phenotypic response to the CAF diet in LEW and WKY rats was assessed by determining the plasma levels of several metabolites previously described as biomarkers of metabolic homeostasis.

Genetic-dependent differences were observed for the modulation of plasma levels of metabolic biomarkers in the rat. Interestingly, although CAF induced a significant increase in the plasma levels of glucose, triglycerides, NEFAs, and $\beta$-hydroxybutyrate in LEW rats, no dietary effects were observed on the plasma levels of these biomarkers in WKY rats except for $\beta$-hydroxybutyrate (Fig. 3A, B, C and F). Furthermore, LEW rats showed higher circulating levels of NEFAs with both analysed diets, whereas the glucose and triglyceride levels were only significantly higher when comparing CAF-fed LEW and WKY rats. Moreover, the $\beta$-hydroxybutyrate plasma levels of STD-fed WKY rats were strikingly higher than those of CAF-fed WKY rats and were also significantly higher than those of STD-fed LEW rats.

The plasma leptin concentration was enhanced by the CAF diet, and although the diet-induced hyperleptinaemia was independent of the genetic background, the leptin concentrations were, for the different proposed diets, significantly higher for LEW rats than for WKY rats (Fig. 3E).

\section{The effects of genotype on the central and peripheral regulation of diet-induced leptin signalling}

The results obtained regarding the phenotypic differential response to diet-induced obesity between LEW and WKY rats pointed to the diet-induced modulation of leptin and its signalling pathway as a possible divergent point between the two strains.

To assess whether the differential phenotypic response to diet-induced obesity in LEW and WKY rats was a consequence of a differential regulation of the leptin signalling pathway genes, the expression of key genes involved in the regulation of leptin signal transduction in relevant organs, including the mesenteric fat pad, the hypothalamus and the liver was measured.

While rats of both genotypes react to CAF diet by inducing the expression of leptin (Lep) in the mesenteric fat pad (Fig. 4A), the expression pattern of the genes involved in the transduction of leptin signalling in the hypothalamus (Fig. 4C) was different. Thus, although no diet-induced effects were observed for the gene expression of leptin receptor B (LepRb) and Stat3, the modulation of Socs 3 gene expression was inversely modulated by the CAF diet in LEW and WKY rats. Therefore, the CAF diet promoted an increase in Socs3 gene expression in LEW rats and a strong inhibition of Socs 3 gene expression in WKY rats.

Furthermore, the diet-induced modulation of leptin receptor $\mathrm{B}$ gene expression in the liver was also opposite for LEW and WKY rats; in LEW rats, the gene expression of $L e p R b$ was upregulated, and in WKY rats, it was not (Fig. 4B). Nonetheless, although Socs3 gene expression in the liver was downregulated by the CAF diet in both strains, this inhibition was only significant in LEW rats.
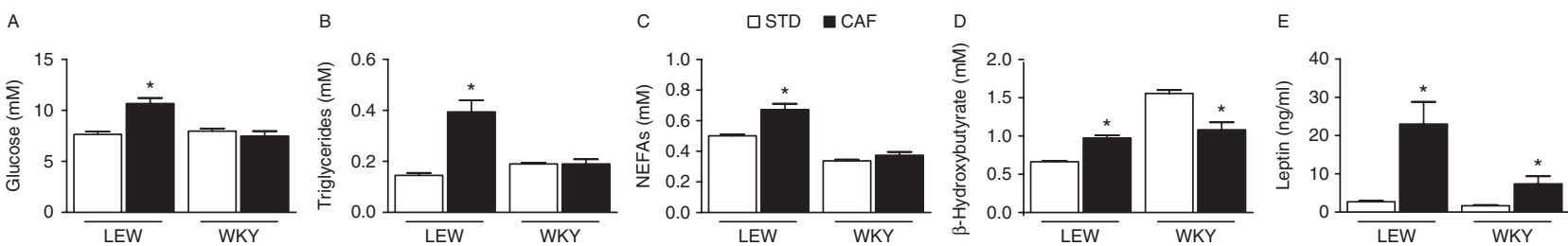

\section{Figure 3}

Circulating levels of the biomarkers involved in dietary responsiveness. The plasma concentration of glucose (A) (standard two-way ANOVA; $n=5 /$ group, $P=0.008$ for diet, $P=0.004$ for strain and $P=0.001$ for diet and strain interaction), triglycerides (standard two-way ANOVA; $n=5 /$ group, $P=0.001$ for diet, $P=0.005$ for strain and $P<0.001$ for diet and strain interaction) (B), NEFAs (standard two-way ANOVA; $n=5 /$ group, $P=0.360$ for diet, $P=0.003$ for strain and $P=0.139$ for diet and strain interaction) (C), $\beta$-hydroxybutyrate (D) (standard two-way ANOVA; $n=5 /$ group, $P=0.002$ for diet, $P=0.010$ for strain and $P<0.001$ for diet and strain interaction) and leptin (E) (standard two-way ANOVA; $n=5 /$ group, $P=0.01$ for diet, $P=0.041$ for strain and $P=0.072$ for diet and strain interaction) were measured in STD- and CAF-fed LEW and WKY rats. The data represent the mean \pm S.E.M. ${ }^{*} P<0.05$ between the rats fed with the STD diet and the CAF diet within each strain (the diet effect).

Published by Bioscientifica Ltd. 

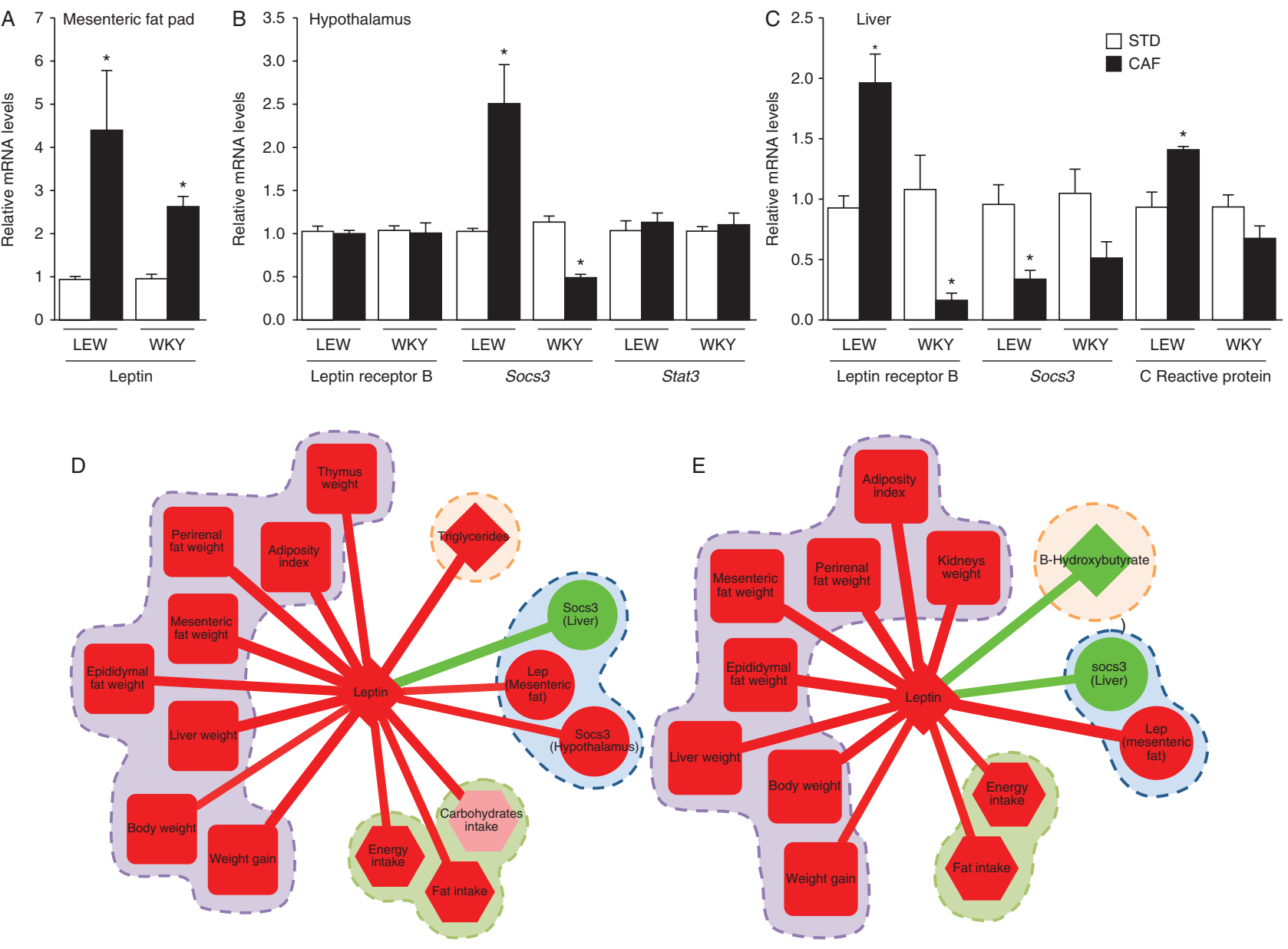

$\mathrm{F}$

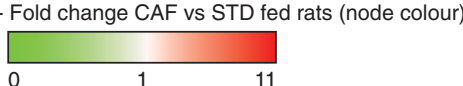

Spearman $r$ (edge colour)

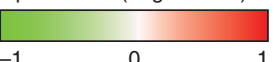

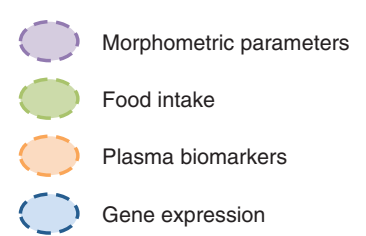

Gene expression

\section{Figure 4}

The expression of key genes involved in the regulation of leptin signalling. Gene expression was measured by RT-PCR. (A) The effect of the CAF diet on leptin gene expression in mesenteric fat pads from LEW and WKY rats. (B) The effect of diet on the expression of leptin receptor B, Socs3 and C-reactive protein in the liver. (C) The effect of diet on the hypothalamic regulation of leptin receptor $B$ and Socs3 gene expression in LEW and WKY rats. ${ }^{*} P<0.05$ between the rats fed with the STD diet and the CAF diet within each strain (diet effect). (D) Leptin signal transduction and SOCS3mediated signal attenuation. Leptin recognition by leptin receptor $B$ in the hypothalamus leads to the activation of JAK2 tyrosine kinase, leading to the autophosphorylation of tyrosine residues on JAK2 and the phosphorylation of $\mathrm{Tyr}_{985}$ and $\mathrm{Tyr}_{1138}$, on the intracellular tail of LepRb. The phosphorylation of LepRb Tyr ${ }_{1138}$ recruits and activates STAT3, leading

The CAF-mediated modulation of $\mathrm{Crp}$ gene expression in the liver was also determined as a biomarker of dietinduced inflammation. The gene expression of Crp was only significantly upregulated by the CAF diet in LEW rats. Printed in Great Britain to its nuclear translocation and the induction of SOCS3 gene expression. SOCS3 acts as a negative-feedback inhibitor of leptin signalling by binding directly to JAK2 and LepRb Tyr ${ }_{985 .}$ ( $E$ and F) Association between leptin and biomarkers related with the development and progression of obesity. The network of significant non-parametric Spearman correlations $(P<0.05)$ between the circulating leptin levels and morphometric parameters, food intake-related values, circulating metabolites and the expression of the genes involved in leptin signalling in LEW and WKY rats respectively. The node colour represents the fold change between the levels of the corresponding parameter in CAF and STD-fed LEW and WKY rats; red nodes correspond to parameters that are upregulated by CAF diet. The edge colour represents the significant Spearman correlation relationship.

Finally, to analyse the role of leptin in the differential phenotypic response to the CAF diet in LEW and WKY rats, the direct relationship between circulating leptin levels and the metabolic-related traits was determined.

Published by Bioscientifica Ltd 
When comparing the significant relationships between plasma leptin levels and the measured metabolic traits, different diet-specific and genotype-specific effects were observed (Fig. 4E and F). Strong relationships were observed between circulating leptin levels and the measured levels of energy and fat intake, final body weight, weight gain, the expression of Lep in mesenteric fat and the expression of Socs 3 in the liver for both genetic backgrounds, suggesting that diet is the main modulator of these effects. However, the relatively high correlations observed between plasma leptin levels and thymus weight, carbohydrate intake, the expression of Socs 3 in the hypothalamus and the circulating levels of triglycerides in LEW rats and the correlation between plasma leptin and $\beta$-hydroxybutyrate in WKY rats also suggest strainspecific effects.

\section{Discussion}

In the present study, we showed the differential phenotypic response of two homogeneous genetic backgrounds, LEW and WKY rats, subjected to a metabolic challenge based on a diet with high-energy density. We report how the CAF diet resulted in an increase in weight gain due to diet-induced hyperphagia and to the hedonic preference for fat intake, reflected primarily in the increased adiposity, independent of the strain (i.e., genetic background). The sequence similarity between both strains is around $25 \%$.

Despite the fact that both genetic backgrounds showed hyperphagia and increased adiposity in response to the CAF diet challenge, the postprandial energy metabolism pattern and the substrate utilisation by peripheral organs were different for both strains. The CAF diet-induced body weight increase of LEW rats was supported not only by the increase in the energy intake but also by the maintenance of the energy expenditure, as well as by the switch to fat instead of carbohydrates as the primarily oxidation source to obtain energy. This pattern of energy metabolism is consistent with the typical energetic response, which was previously observed in outbred rats in response to a CAF diet (Spiegelman \& Flier 2001). On the other hand, the lower metabolic rate observed in STD-fed WKY rats may be attributed to an ineffective use of carbohydrates as the major energy substrate, resulting instead in the $\beta$-oxidation of fatty acids as the principal fuel source. In fact, some degree of carbohydrate intolerance had been reported previously for lean WKY rats, although on that occasion rats were still not fully inbred (Ikeda et al. 1981, Kava et al. 1990). Furthermore, the very high levels of circulating $\beta$-hydroxybutyrate, one of the main ketone bodies produced by the liver, determined in the plasma of STDfed WKY rats supported this non-predominant oxidative glucose utilisation (McGarry \& Foster 1980, Laffel 1999). Moreover, the disparity observed between the circulating levels of ketone bodies in STD- and CAF-fed WKY rats may be an indication that the source of the dietary carbohydrates plays a key role in carbohydrate metabolism, as has been previously described for other rat strains (Michaelis et al. 1986). Therefore, although no effect of diet on the amount of carbohydrate intake was observed, the complexity of the major carbohydrate form found in each type of diet was different. Thus, while the main carbohydrate found in the STD diet was starch, a polysaccharide, the most abundant carbohydrate found in the CAF diet was sucrose, a disaccharide.

Plasma metabolic profiling also corroborated the disparity in the phenotypic response to CAF diet of both genetic backgrounds. Thus, while CAF diet challenge induced hyperglycaemia and hypertriglyceridaemia in LEW rats, no effect of diet was observed in WKY rats. Furthermore, although an increase in leptin circulating levels was observed independently of the genetic background, the downstream response to hyperleptinaemia was strain-specific. Therefore, in LEW rats, the strong relationship between plasma leptin levels and the nutritional statusassociated traits, including the food intake, adiposity and plasma triglycerides, may be associated with the metabolic phenotype resulting from central leptin resistance, a pivotal event underlying the development of obesity and its associated dysfunctions (Zhang \& Scarpace 2006). The secreted leptin levels showed close relationships with dietinduced traits involved in the upstream regulation of leptin signalling such as food intake and adiposity in WKY rats. However, the metabolic traits that were influenced by leptin signalling, such as triglycerides levels, did not respond to a diet-induced increase in adiposity. Thus, in WKY rats, the diet-induced hyperleptinaemia was not reflected in an impairment of lipid metabolism. This finding can have important implications because of the role of leptin as an integral endocrine regulator of energy metabolism. Therefore, our data suggest continuous functional interactions between the leptin axis and other metabolic pathways, including the lipid and glucose metabolism pathways. Leptin is involved, among other functions, in fat storage regulation and in the modulation of the glucose metabolism independent of its effects on energy balance, mediated by the regulation of fatty acid $\beta$-oxidation (Minokoshi et al. 2002, Unger 2003) or control of peripheral tissue insulin sensitivity (Morton \& Schwartz 2011).

Published by Bioscientifica Ltd. 
Taken together all the phenotypic results on the differential response of LEW and WKY rats to a dietinduced energy surplus indicate that the regulation of the leptin signalling axis might underlie the strain-specific response to CAF diet. Our data confirm, in a genotypeindependent manner, what has been previously hypothesized: an overfeeding-induced increase in adiposity promotes the adipocyte-mediated upregulation of leptin transcription, increasing the circulating leptin levels (Frederich et al. 1995b). Once it is secreted, plasma leptin can be sensed by the central nervous system, and more specifically by the hypothalamus, where leptin recognition by its receptor (LepRb) elicits the JAK2-mediated activation of STAT3, a transcription factor that is essential for leptin functionality. Our mRNA expression analysis of the genes involved in the leptin receptor signal transduction in the hypothalamus revealed important differences in the regulation of downstream leptin signalling between LEW and WKY rats. Although no diet-specific effects were observed regarding the relative expression of $L e p R b$ and Stat3 in the hypothalamus, the modulation of hypothalamic Socs3 expression was dependent not only on the diet but also on the genetic background. This strain-specific regulation of Socs 3 expression by the CAF diet is highly relevant for the functionality of leptin signalling because Socs 3 acts as a negative-feedback regulator of leptin signal transduction (Mori et al. 2004). Thus, while the enhanced expression of Socs 3 in the hypothalamus of CAF-fed LEW rats confirms the attenuation of central leptin signalling, a hallmark feature of leptin resistance (Knobelspies et al. 2010, Olofsson et al. 2013), the downregulation of Socs3 expression observed in CAF-fed WKY rats is associated with an increase in central leptin sensitivity. Therefore, the hyperleptinaemia in WKY rats was not translated to an attenuation of the leptin signalling, but the opposite. Our findings can have potential health implications because the suppression of hypothalamic Socs 3 expression has been proposed as a potential therapy to confer resistance to diet-induced obesity (Mori et al. 2004).

Furthermore, the effect of diet on the peripheral regulation of leptin signalling was also strain-specific, and the impairment of central leptin signalling in CAF-fed LEW rats was also observed in the liver by the significant upregulation of $L e p R b$ transcription and the downregulation of Socs3, a phenomenon that is associated with an increase in liver insulin sensitivity and with the promotion of the lipogenesis-induced inflammatory response and the obesity (Sachithanandan et al. 2010). Nonetheless, in CAF-fed WKY rats, LepRb gene expression was downregulated by the diet, whereas Socs3 expression was slightly but not significantly decreased. This peripheral inactivation of $L e p R b$ in CAF-fed animals compared with the STD-fed ones is consistent with the over-repression of Socs 3 in the hypothalamus and is supported by previous results (Olofsson et al. 2013).

Our data proposed SOCS3 and its differential regulation as an important underlying event in the genotypedependent responsiveness to the dietary energy density observed between LEW and WKY rats. In fact, the molecular mechanisms that underlie the SOCS3-mediated attenuation of leptin intracellular signalling are based on the binding of SOCS3 to phosphorylated $\mathrm{Tyr}_{985}$ in the leptin receptor and the direct binding of SOCS3 to JAK2, blocking its docking domain and preventing its phosphorylation as well as the phosphorylation and then the activation of STAT3 (Bjørbæk et al. 1999, El-Haschimi et al. 2000). We can therefore hypothesize that the maintenance of STAT3 activation through its phosphorylation consequence of SOCS3 is an essential step for the phenotypic response to diet. Moreover, $L e p R b$ and its intracellular tyrosine residues may also play a pivotal role in the regulation of STAT3 activation under chronic leptin stimulation (Knobelspies et al. 2010).

\section{Conclusion}

In summary, our integrated genetic and phenotypic analyses in LEW and WKY rats subjected to metabolic challenge show that the leptin axis, and specifically the LepRb-mediated regulation of STAT3 activation under conditions of chronic ligand stimulation, plays a pivotal role in the genotype-dependent adaptation to dietinduced obesity.

\section{Declaration of interest}

The authors declare that there is no conflict of interest that could be perceived as prejudicing the impartiality of the research reported.

\section{Funding}

This study was supported by a grant (AGL2014-55347) from the Ministerio de Ciencia e Innovación of Spanish Government (MICCIN). E P acknowledges funding from the Medical Research Council. N M holds a PhD grant from the University Rovira i Virgili and a mobility grant from Ministerio de Educación.

\section{References}

Arch JRS, Hislop D, Wang SJY \& Speakman JR 2006 Some mathematical and technical issues in the measurement and interpretation of open-circuit indirect calorimetry in small animals. International Journal of Obesity $\mathbf{3 0}$ 1322-1331. (doi:10.1038/sj.ijo.0803280)

Published by Bioscientifica Ltd. 
Behmoaras J, Bhangal G, Smith J, McDonald K, Mutch B, Lai PC, Domin J, Game L, Salama A, Foxwell BM et al. 2008 Jund is a determinant of macrophage activation and is associated with glomerulonephritis susceptibility. Nature Genetics 40 553-559. (doi:10.1038/ng.137)

Behmoaras J, Smith J, D'Souza Z, Bhangal G, Chawanasuntoropoj R, Tam FWK, Pusey CD, Aitman TJ \& Cook HT 2010 Genetic loci modulate macrophage activity and glomerular damage in experimental glomerulonephritis. Journal of the American Society of Nephrology $\mathbf{2 1}$ 1136-1144. (doi:10.1681/ASN.2009090968)

Bjørbæk C, El-Haschimi K, Frantz JD \& Flier JS 1999 The role of SOCS-3 in leptin signaling and leptin resistance. Journal of Biological Chemistry 274 30059-30065. (doi:10.1074/jbc.274.42.30059)

Bouchard C 2008 Gene-environment interactions in the etiology of obesity: defining the fundamentals. Obesity 16 S5-S10. (doi:10.1038/ oby.2008.528)

D'Souza Z, McAdoo SP, Smith J, Pusey CD, Cook HT, Behmoaras J \& Aitman TJ 2013 Experimental crescentic glomerulonephritis: a new bicongenic rat model. Disease Models \& Mechanisms 6 1477-1486. (doi:10.1242/dmm.012328)

El-Haschimi K, Pierroz DD, Hileman SM, Bjørbaek C \& Flier JS 2000 Two defects contribute to hypothalamic leptin resistance in mice with diet-induced obesity. Journal of Clinical Investigation 105 1827-1832. (doi:10.1172/JCI9842)

Ferrannini E 1988 The theoretical bases of indirect calorimetry: a review. Metabolism: Clinical and Experimental 37 287-301. (doi:10.1016/ 0026-0495(88)90110-2)

Frederich RC, Löllmann B, Hamann A, Napolitano-Rosen A, Kahn BB, Lowell BB \& Flier JS 1995a Expression of ob mRNA and its encoded protein in rodents. Impact of nutrition and obesity. Journal of Clinical Investigation 96 1658. (doi:10.1172/JCI118206)

Frederich RC, Hamann A, Anderson S, Löllmann B, Lowell BB \& Flier JS $1995 b$ Leptin levels reflect body lipid content in mice: evidence for diet-induced resistance to leptin action. Nature Medicine 1 1311-1314. (doi:10.1038/nm1295-1311)

Fröhlich M, Imhof A, Berg G, Hutchinson WL, Pepys MB, Boeing H, Muche R, Brenner H \& Koenig W 2000 Association between C-reactive protein and features of the metabolic syndrome: a population-based study. Diabetes Care 23 1835-1839. (doi:10.2337/diacare.23.12.1835)

Galic S, Oakhill JS \& Steinberg GR 2010 Adipose tissue as an endocrine organ. Molecular and Cellular Biochemistry 316 129-139. (doi:10.1016/ j.mce.2009.08.018)

Hu CC, Qing K \& Chen Y 2004 Diet-induced changes in Stearoyl-CoA desaturase 1 expression in obesity-prone and -resistant mice. Obesity Research 12 1264-1270. (doi:10.1038/oby.2004.160)

Ikeda H, Shino A, Matsuo T, Iwatsuka H \& Suzuoki Z 1981 A new genetically obese-hyperglycemic rat (Wistar fatty). Diabetes 30 1045-1050. (doi:10.2337/diab.30.12.1045)

Kanneganti T-D \& Dixit VD 2012 Immunological complications of obesity. Nature Immunology 13 707-712. (doi:10.1038/ni.2343)

Kava R, Peterson RG, West DB \& Greenwood MRC 1990 Wistar diabetic fatty rat. ILAR Journal 32 9-13. (doi:10.1093/ilar.32.3.9)

Knobelspies H, Zeidler J, Hekerman P, Bamberg-Lemper S \& Becker W 2010 Mechanism of attenuation of leptin signaling under chronic ligand stimulation. BMC Biochemistry 11 2. (doi:10.1186/1471-2091-11-2)

Koza RA, Nikonova L, Hogan J, Rim J-S, Mendoza T, Faulk C, Skaf J \& Kozak LP 2006 Changes in gene expression foreshadow diet-induced obesity in genetically identical mice. PLoS Genetics 2 e81. (doi:10.1371/journal. pgen.0020081)

La Cava A \& Matarese G 2004 The weight of leptin in immunity. Nature Reviews Immunology 4 371-379. (doi:10.1038/nri1350)

Laffel L 1999 Ketone bodies: a review of physiology, pathophysiology and application of monitoring to diabetes. Diabetes Metabolism Research and Reviews 15 412-426. (doi:10.1002/(SICI)1520-7560(199911/12) 15:6<412::AID-DMRR72>3.0.CO;2-8)

Maratou K, Behmoaras J, Fewings C, Srivastava P, D'Souza Z, Smith J, Game L, Cook T \& Aitman T 2011 Characterization of the macrophage transcriptome in glomerulonephritis-susceptible and -resistant rat strains. Genes and Immunity 12 78-89. (doi:10.1038/gene.2010.61)

McGarry JD \& Foster DW 1980 Regulation of hepatic fatty acid oxidation and ketone body production. Annual Review of Biochemistry 49 395-420. (doi:10.1146/annurev.bi.49.070180.002143)

Michaelis OE, Ellwood KC, Tulp OL \& Greenwood MRC 1986 Effect of feeding sucrose or starch diets on parameters of glucose tolerance in the LA/N-corpulent rat. Nutrition Research 6 95-99. (doi:10.1016/S02715317(86)80203-2)

Minokoshi Y, Kim Y-B, Peroni OD, Fryer LGD, Müller C, Carling D \& Kahn BB 2002 Leptin stimulates fatty-acid oxidation by activating AMP-activated protein kinase. Nature 415 339-343. (doi:10.1038/ 415339a)

Mori H, Hanada R, Hanada T, Aki D, Mashima R, Nishinakamura H, Torisu T, Chien KR, Yasukawa H \& Yoshimura A 2004 Socs3 deficiency in the brain elevates leptin sensitivity and confers resistance to diet-induced obesity. Nature Medicine 10 739-743. (doi:10.1038/ nm1071)

Morton GJ \& Schwartz MW 2011 Leptin and the central nervous system control of glucose metabolism. Physiological Reviews 91 389-411. (doi:10.1152/physrev.00007.2010)

Myers MG, Cowley MA \& Münzberg H 2008 Mechanisms of leptin action and leptin resistance. Annual Review of Physiology 70 537-556. (doi:10.1146/annurev.physiol.70.113006.100707)

Olofsson LE, Unger EK, Cheung CC \& Xu AW 2013 Modulation of AgRP-neuronal function by SOCS3 as an initiating event in diet-induced hypothalamic leptin resistance. PNAS 110 E697-E706. (doi:10.1073/pnas.1218284110)

Ordovas JM 2008 Genotype-phenotype associations: modulation by diet and obesity. Obesity 16 S40-S46. (doi:10.1038/oby.2008.515)

Parks BW, Nam E, Org E, Kostem E, Norheim F, Hui ST, Pan C, Civelek M, Rau CD, Bennett BJ et al. 2013 Genetic control of obesity and gut microbiota composition in response to high-fat, high-sucrose diet in mice. Cell Metabolism 17 141-152. (doi:10.1016/j.cmet.2012.12.007)

Pérusse L \& Bouchard C 2000 Gene-diet interactions in obesity. American Journal of Clinical Nutrition 72 (5 Supplement) 1285s-1290s.

Sachithanandan N, Fam BC, Fynch S, Dzamko N, Watt MJ, Wormald S, Honeyman J, Galic S, Proietto J, Andrikopoulos S et al. 2010 Liver-specific suppressor of cytokine signaling-3 deletion in mice enhances hepatic insulin sensitivity and lipogenesis resulting in fatty liver and obesity. Hepatology 52 1632-1642. (doi:10.1002/hep. 23861)

Sampey BP, Vanhoose AM, Winfield HM, Freemerman AJ, Muehlbauer MJ, Fueger PT, Newgard CB \& Makowski L 2011 Cafeteria diet is a robust model of human metabolic syndrome with liver and adipose inflammation: comparison to high?fat diet Obesity 19 1109-1117. (doi:10.1038/oby.2011.18)

Speakman JR 2004 Obesity: the integrated roles of environment and genetics. Journal of Nutrition 134 (8 Supplement) 2090S-2105S.

Spiegelman BM \& Flier JS 2001 Obesity and the regulation of energy balance. Cell 104 531-543. (doi:10.1016/S0092-8674(01)00240-9)

Unger RH 2003 The physiology of cellular liporegulation. Annual Review of Physiology 65 333-347. (doi:10.1146/annurev.physiol.65.092101. 142622)

Zhang Y \& Scarpace PJ 2006 The role of leptin in leptin resistance and obesity. Physiology \& Behavior 88 249-256. (doi:10.1016/j.physbeh. 2006.05.038)

Received in final form 14 September 2015

Accepted 8 October 2015

Accepted Preprint published online 8 October 2015 http://jme.endocrinology-journals.org

DOI: 10.1530/JME-15-0089
๑) 2016 Society for Endocrinology Printed in Great Britain
Published by Bioscientifica Ltd 